\title{
THE NONLINEARLY DAMPED OSCILLATOR
}

\author{
JUAN LUIS VÁZQUEZ ${ }^{1}$
}

\begin{abstract}
We study the large-time behaviour of the nonlinear oscillator

$$
m x^{\prime \prime}+f\left(x^{\prime}\right)+k x=0,
$$

where $m, k>0$ and $f$ is a monotone real function representing nonlinear friction. We are interested in understanding the long-time effect of a nonlinear damping term, with special attention to the model case $f\left(x^{\prime}\right)=A\left|x^{\prime}\right|^{\alpha-1} x^{\prime}$ with $\alpha$ real, $A>0$. We characterize the existence and behaviour of fast orbits, i.e., orbits that stop in finite time.
\end{abstract}

Mathematics Subject Classification. 34C15.

Received September 18, 2002. Revised November 29, 2002.

\section{INTRODUCTION}

We study the behaviour of the nonlinearly damped oscillator

$$
m x^{\prime \prime}(t)+f\left(x^{\prime}(t)\right)+k x(t)=0
$$

where $m, k>0$ are constants and $f$ is a nonlinear real function, representing nonlinear friction or other damping effects. We are interested in understanding the long-time effect of a nonlinear damping term on the stabilization of the trajectories. Special attention is given to stabilization in finite time, i.e., to the existence of so-called fast orbits. If $f(s)$ is monotone near $s=0$, the existence of such orbits is completely characterized in terms of $f$, cf. Theorem 4.3 .

The equation with linear damping, i.e., $f\left(x^{\prime}\right)=A x^{\prime}$ with $A>0$, appears in all ODE textbooks and leads to decay towards $x=0$ with an exponential rate in time, according to three different possibilities. The alternative depends on the relative size of $A$ with respect to $m$ and $k$. More precisely, there is a critical value $A_{0}=(4 m k)^{1 / 2}$, and the values $A<A_{0}$ are known as subcritical damping, those with $A>A_{0}$ as supercritical or overdamping, $c f$. [17]; the critical value $A_{0}$ corresponds to a double root of the associated characteristic polynomial, $p(r)=m r^{2}+A r+k$. In the subcritical case solutions decay to zero after infinitely many oscillations since the origin is a stable focus in phase plane (phase planes are carefully explained and analyzed below). In the two other cases, at most a change of sign is allowed (a stable node). We recall that for $A=0$ there is no damping and the picture in phase plane is a center, while for $A<0$ the origin is an unstable point. Therefore, the stabilization problem is not posed in those cases.

Keywords and phrases: Nonlinear oscillator, nonlinear damping, fast orbits.

1 Departamento de Matemáticas, Universidad Autónoma de Madrid, 28049 Madrid, Spain; e-mail: juanluis.vazquez@uam.es 
In order to follow a path of increasing difficulty and obtain information as detailed as possible in the process, we devote special attention to the power-like case

$$
f(s)=A|s|^{\alpha-1} s, \quad A>0
$$

where the exponent $\alpha$ can be any real number. Dividing by $m$ we write the equation as

$$
x^{\prime \prime}(t)+a\left|x^{\prime}\right|^{\alpha-1} x^{\prime}+b x=0,
$$

with $b=k / m$ and $a=A / m$. We have mentioned the case $\alpha=1$. It is easily seen by linearization that when $\alpha>1$, the behaviour of this equation for small $x^{\prime}$ is essentially the same as in the subcritical case, so that stabilization to zero takes place in infinite time and it happens with infinitely many oscillations in forward time.

This leaves the exponents $\alpha<1$ as interesting cases to study. The case $\alpha=0$, known as Coulomb friction, is well known in control theory and can be explicitly integrated [19]. The study below allows to observe a new and interesting nonlinear decay behaviour, namely, complete stop in finite time: there is a time $t_{0}<\infty$ such that $x\left(t_{0}\right)=x^{\prime}\left(t_{0}\right)=0$. Moreover, there is one orbit with $x\left(t_{0}\right)=0$ and the rest of orbits stop at different final locations, see below Section 3 .

In the case $\alpha \in(0,1)$ it was proved by Haraux [16] that orbits $\left(x(t), x^{\prime}(t)\right)$ go to $(0,0)$ as $t$ increases. This case has been further studied by Díaz and Liñán [10] in a recent paper appeared in the journal of the Spanish Academy of Sciences. The authors establish the existence of orbits with complete stop in finite time and orbits with decay in infinite time with an exponential rate, and this happens for all $\alpha$ in that range. However, though the formal analysis gives a clear picture, the authors state the rigorous analysis as an open problem and exhibit partial progress towards that goal using super- and subsolution estimates.

This note contains a complete rigorous analysis of all cases $\alpha<1$. Actually, our study is based on two standard tools of dynamical systems: phase-plane analysis and blow-up to resolve the singularities. In the main application of the method we arrive at a singularity with a normal form which happens to be a saddle as expected. This establishes the existence and uniqueness of a distinguished orbit, that we call the fast orbit, representing complete stop in finite time. The rest of the orbits move locally away from this behaviour. A study of phase plane leads on one side of the fast orbit to orbits that change sign (oscillations), and on the other side to slow orbits that come to rest in infinite time with no oscillation for large times. Regularity is shown and asymptotic expansions are obtained and justified.

The analysis is extended past $\alpha=0$ to the case of singular friction $\alpha \leq 0$, where stabilization at $x=0$ takes place only along the fast orbit. The rest of the orbits stabilize in finite time to points $x_{\infty} \neq 0$.

Afterwards, we turn our attention to a general case where $f$ is not necessarily power-like. Assuming as basic assumptions that $f(v)$ is continuous and $C^{1}$ smooth for $v \neq 0$, that $f(v) v>0$ for $v \neq 0$, and $f$ is monotone near $v=0$, we obtain a necessary and sufficient condition on $f$ for the existence of a fast orbit. The condition consists of the integrability at $s=0+$ or $0-$ of the function $1 / f(s), c f$. Theorem 4.3 and the rest of Section 4 . Monotonicity of $f(s)$ is required only for small values of $s$, and it can be replaced by even weaker conditions. As for the basic assumptions, we remark that in the application to a spring subject to friction it is natural to assume that the friction force is a monotone function of the speed. So the characterization leaves out only "pathological forms" of $f$, but mathematically these forms do exist.

In order to give the reader some insight into related mathematical questions, we add a section where the necessary and sufficient condition is compared to the similar solution to the question about finite traveling waves and finite propagation, which is a main issue in nonlinear diffusion. The origin of the similarity is explained. Before proceeding with the proofs, the motivation coming from nonlinear wave equations is discussed. This completes our purpose in the paper. 


\subsection{Motivation from wave equations}

The present investigation was started by the author as a first step with a view to understanding the behaviour of a damped string,

$$
u_{t t}+f\left(u_{t}\right)-c^{2} u_{x x}=0
$$

To our knowledge, this problem remains open. But it may be interesting to note that our analysis applies when we consider the simpler model

$$
u_{t t}-c^{2} u_{x x}+\left\|u_{t}\right\|_{2}^{p-1} u_{t}=0
$$

where the norm $\left\|u_{t}\right\|_{2}$ is taken for every fixed $t$ in $L^{2}(\mathbb{R})$. If we take as space domain $I=(0, a)$ and look for solutions which solve the problem with zero Dirichlet boundary data, we may try the Ansatz

$$
u(x, t)=\sin (\omega x) X(t)
$$

with $\omega=\pi / a$. In that situation $X(t)$ must solve our original equation in the form

$$
X^{\prime \prime}+k X+d\left|X^{\prime}\right|^{p-1} X^{\prime}=0
$$

with $k=c^{2} \pi^{2} / a^{2}$ and $d=\left(\int_{0}^{a} \sin ^{2}(\omega x) \mathrm{d} x\right)^{(p-1) / 2}=(a / 2)^{(p-1) / 2}$. We get complete stop of the string in finite time for a class of initial configurations when $p<1$. The result can be extended to friction terms of the form $g\left(\left\|u_{t}\right\|_{2}\right) u_{t}$ with suitable $g$. We leave the translation of our results of Section 4 to the reader.

\section{The PHASE Plane}

Let us fix $\alpha$ between 0 and 1. We first prepare the problem. Following standard practice, we introduce the velocity variable $v=-x^{\prime}$ and write equation (1.1) as a system in the form

$$
\left\{\begin{array}{l}
\frac{\mathrm{d} x}{\mathrm{~d} t}=-v \\
\frac{\mathrm{d} v}{\mathrm{~d} t}=-a|v|^{\alpha-1} v+b x
\end{array}\right.
$$

Dividing both equations we get

$$
v \frac{\mathrm{d} v}{\mathrm{~d} x}=a|v|^{\alpha-1} v-b x
$$

We observe that the study of the phase plane is trivial in the quadrants Q-II, $\{(x, v): x<0, v>0\}$, and Q-IV, $\{(x, v): x>0, v<0\}$, where no approach to the origin $(0,0)$ takes place since the sign of the friction term $\left(-a|v|^{p-1} v\right)$ helps the sign of the elastic term $(b x)$ in forcing the solutions to go past $x=0$. This is a well-known argument that we will explain later in some detail in the case of general $f$, see Section 4.2 . Moreover, by symmetry $x \mapsto-x, v \mapsto-v$, we can reduce quadrant Q-III to the first. Hence, only quadrant Q-I $=\{(x, v): x>0, v>0\}$ need be studied. In this case we may write the equation as

$$
v \frac{\mathrm{d} v}{\mathrm{~d} x}=a v^{\alpha}-b x, \quad \text { with } \quad x, v>0 .
$$




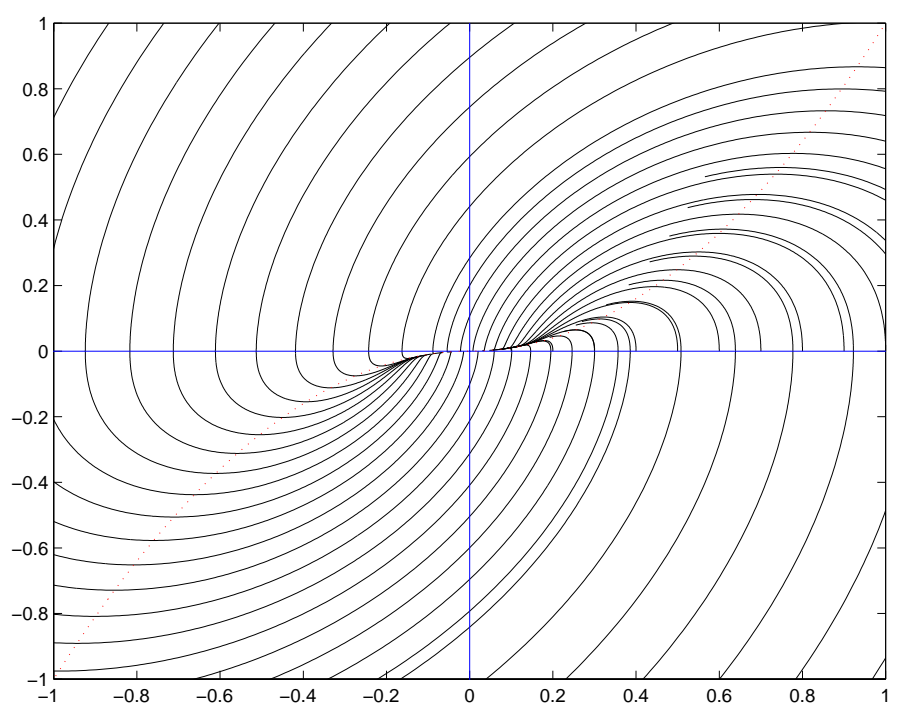

Figure 1 . The $(x, v)$ phase plane for $\alpha=1 / 2$. The dotted line the 0 -cline.

The behaviour of solutions near the steady state, $x=v=0$, is described in the following result:

Theorem 2.1. For $0<\alpha<1$ all orbits of system (2.1) have a finite number of sign changes in their positive evolution. Indeed, there are three types of orbits in the first quadrant:

(i) a unique fast orbit $\Gamma_{*}$ that goes to zero in finite time;

(ii) infinitely many slow orbits that go to zero in infinite time. Precisely, the ones located under $\Gamma_{*}$;

(iii) infinitely many changing sign orbits. Precisely, the ones located over $\Gamma_{*}$.

These options are characterized by the relation between $v$ and $x^{1 /(2-\alpha)}$ along orbits in the first quadrant. The limit $\lim _{x \rightarrow 0} v(x) / x^{1 /(2-\alpha)}$ admits three values: a constant $0<c<\infty$ in option (i), 0 in option (ii), infinity in option (iii). The value of the constant in the first case is $c=((2-\alpha) a)^{1 /(2-\alpha)}$.

Remarks. (i) A symmetric situation happens in the third quadrant, with $x<0$ and $v=-x^{\prime}<0$. The curve $\Gamma_{*}$ and its symmetric $\Gamma_{* *}$ can be prolonged back in time to form the global fast manifold, $\Gamma$.

(ii) In the two first types $x(t)$ and $x^{\prime}(t)$ have one sign, either positive or negative; in the third type, $x(t)$ oscillates a number of times until it is captured into the fast orbit or the slow orbits (the latter being more probable). This is easy to see from the pictures and the observation that solutions go eventually to $(0,0)$.

(iii) The rate of decay of the slow orbit is $v(x) \sim x^{1 / \alpha}$, see (2.14). This implies a power decay of $x(t)$ as $t \rightarrow \infty$ like $t^{-\mu}$ with $\mu=\alpha /(1-\alpha)$, see (2.15). In comparison with the fast orbit, note that $1 /(2-\alpha)<1 / \alpha$ for $\alpha<1$.

Let us now proceed with the analysis and proof of the theorem.

Fast orbit. Our analysis begins with the formal computation for the fast orbit. Only the inertial and friction terms are taken into account in the end, the elastic term $b x$ turns out to be lower-order, so that $\mathrm{d} v / \mathrm{d} x \sim a v^{\alpha-1}$. In order to normalize the calculation we introduce the variable

$$
w=v^{2-\alpha}
$$

which transforms equation $(2.2)$ into

$$
\frac{\mathrm{d} w}{\mathrm{~d} x}=a_{1}-b_{1} \frac{x}{w^{\beta}}, \quad \beta=\frac{\alpha}{2-\alpha} .
$$


We see that $0<\alpha \leq 1$ translates into $0<\beta \leq 1$. The constants $a_{1}$ and $b_{1}$ are given by $a_{1}=(2-\alpha) a$, $b_{1}=(2-\alpha) b$. In case $\alpha=1$ this is reduced back into the well known linear system, and the analysis produces either a stable focus or a stable node, depending on $a_{1}$ and $b_{1}$, as explained in the Introduction.

Blow-up. The analysis of the case $\alpha<1$ needs another preparatory step (resolution of the singularity) in the form of blow-up. Since the formal analysis produces a (possible) solution of the form $w(x) \sim a_{1} x$, we introduce the blow-up variable

$$
z=\frac{a_{1} x-w}{a_{1} x}
$$

i.e., $w=a_{1} x(1-z)$. We also put $x^{1-\beta}=\xi$. Then, equation (2.2) becomes

$$
x \frac{\mathrm{d} z}{\mathrm{~d} x}=(1-\beta) \xi \frac{\mathrm{d} z}{\mathrm{~d} \xi}=-z+b_{1} \frac{\xi}{a_{1}^{1+\beta}(1-z)^{\beta}},
$$

or equivalently,

$$
\xi \frac{\mathrm{d} z}{\mathrm{~d} \xi}=b_{2} \frac{\xi}{(1-z)^{\beta}}-d_{1} z
$$

with $b_{2}=b_{1} /\left(a_{1}^{1+\beta}(1-\beta)\right)$ and $d_{1}=1 /(1-\beta)$. This is the equation that has to be carefully analyzed as $\xi \rightarrow 0$, $\xi>0$. We can also write it as a system:

$$
\left\{\begin{array}{l}
\frac{\mathrm{d} \xi}{\mathrm{d} s}=-\xi(1-z)^{\beta} \\
\frac{\mathrm{d} z}{\mathrm{~d} s}=-b_{2} \xi+d_{1} z(1-z)^{\beta}
\end{array}\right.
$$

There are two critical points, namely, A: $\xi=0, z=0$ and $\mathrm{B}: \xi=0, z=1$.

The normal form: a saddle. We are now ready for the rigorous theory. It is clear that near $A$ the linearized system has a saddle-point topology, the configuration we are looking for. In fact, the system is (locally) a small and smooth perturbation of

$$
\left\{\begin{array}{l}
\frac{\mathrm{d} \xi}{\mathrm{d} s}=-\xi \\
\frac{\mathrm{d} z}{\mathrm{~d} s}=-b_{2} \xi+d_{1} z
\end{array}\right.
$$

It has two eigenvalues, $\lambda_{1}=d_{1}$ with eigenvector $v_{1}=(0,1)$; and $\lambda_{2}=-1$ with eigenvector $v_{2}=\left(d_{1}+1, b_{2}\right)$.

We are done. The theory $(c f .[15,21])$ says that there is only one orbit entering the origin $(\xi=0, z=0)$ (the stable manifold) with eigenvalue -1 and tangent vector $v_{2}$ at $(0,0)$. It takes infinite time in doing so in the $s$ variable, more precisely it converges in an exponential way; but $\xi$ is finite: $\xi \rightarrow 0$ of course. We call this manifold (curve) $\Gamma_{*}$. 
Summing up the theory, we have:

Theorem 2.2. The stable manifold is given by a $C^{\infty}$ smooth function defined for some interval $0 \leq \xi \leq \xi_{0}$ :

$$
z_{*}(\xi)=a_{2} \xi+a_{3} \xi^{2}+\cdots
$$

where $a_{2}=b_{2} /\left(d_{1}+1\right)$ and the remaining coefficients can also be calculated by formal methods.

This implies that

$$
w_{*}(x)=a_{1} x-a_{1} z_{*}\left(x^{1-\beta}\right) x=a_{1} x-a_{2} x^{2-\beta}+O\left(x^{3-2 \beta}\right),
$$

hence, putting $\gamma=1 /(2-\alpha) \in(1 / 2,1)$,

$$
v_{*}(x)=a_{1}^{\gamma} x^{\gamma}\left(1-z_{*}\left(x^{1-\beta}\right)^{\gamma}=c_{1} x^{\gamma}+c_{2} x^{\gamma+1-\beta}+\cdots,\right.
$$

with $c_{1}=((2-\alpha) a)^{1 /(2-\alpha)}$ as predicted by the formal analysis. Finally, integration of $\mathrm{d} t=-\mathrm{d} x / v$ gives

$$
x_{*}(t)=d\left(t_{0}-t\right)^{(2-\alpha) /(1-\alpha)}\left(1+o\left(t_{0}-t\right)\right)
$$

for some finite $d$ depending on the parameters and $t_{0}$ depending on the initial location, and for $t<t_{0}$. These are the expressions for the unique fast orbit which ends its trajectory in the first quadrant.

There exists a symmetric fast orbit, $\Gamma_{* *}$, given by the expression $x_{* *}(t)=-x_{*}(t)$ with negative amplitudes (eventually). Both $\Gamma_{*}$ and $\Gamma_{* *}$ can be prolonged back in time as far as we want, and they spiral away from zero. Together they form the complete fast orbit $\Gamma$. This is all.

The numerical calculations show the orbits of the nonlinear systems (2.5) and (2.9), corresponding to parameters $\alpha=1 / 2, a=b=1$, hence $\beta=1 / 3$. The latter picture unfolds the singularity of the former at $(0,0)$; it separates the fast orbit (which is now the orbit entering $(0,0)$ ) from the slow orbits (which enter $(0,1)$ ). It clearly displays the fast orbit as the stable manifold near a hyperbolic point of a planar dynamical system.

Change of sign. We now consider the orbits that change sign. They are represented in Figure 2 by the orbits that pass to the second quadrant in the $(x, w)$ plane, i.e., they lie on top of $\Gamma$. This means that $z(\xi)<z_{*}(\xi)$. By the saddle-point structure of Figure 3 around $(0,0)$, these orbits go to minus infinity as $\xi \rightarrow 0$, which means that

$$
\lim _{x \rightarrow 0} \frac{w(x)}{x}=\infty
$$

Now, we know from equation (2.8) that $\mathrm{d} w / \mathrm{d} x \leq a_{1}$. Due to the location in the phase-plane we also have

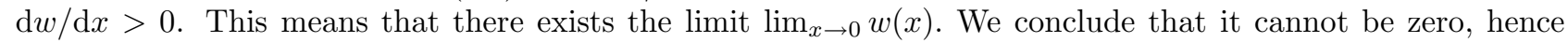
it can only be positive. In other words, all these solutions cross over to the second quadrant and represent solutions that change sign.

Slow orbits. Finally, solutions lying below $w_{*}(x)$ correspond to orbits going up in the $(\xi, z)$ plane. According to Figure 3 the attractor point is $B: \xi=0, z=1$, so that we have

$$
\lim _{x \rightarrow 0} \frac{w(x)}{x}=0
$$

as stated in the theorem. Formal analysis says that the orbit will approach the inertial manifold $a v^{\alpha}=b x$. In order to perform the precise analysis we introduce the change of variables $v^{\alpha}=W=(b / a) x(1+y)$, i.e.,

$$
y=\frac{a v^{\alpha}-b x}{b x} .
$$




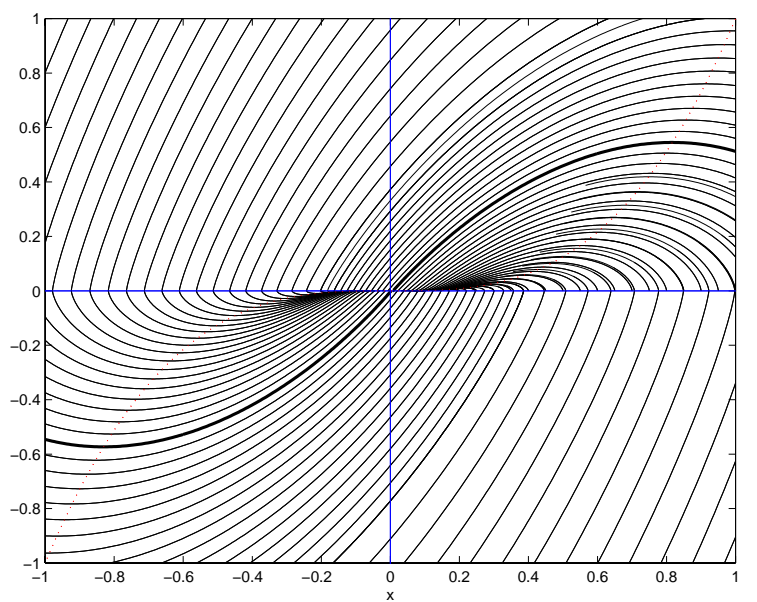

Figure 2. $(x, w)$ phase plane. The thick line is $\Gamma$.

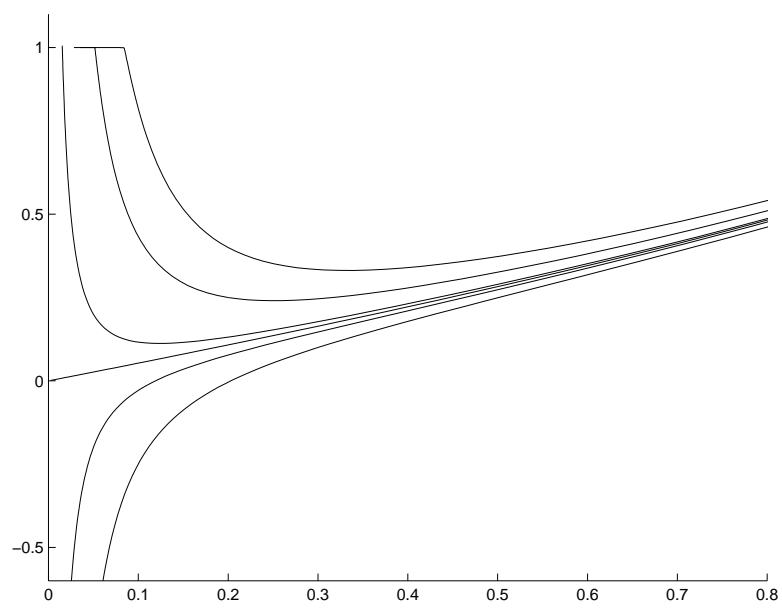

Figure 3. $(\xi, z)$ phase plane.

Then (2.3) becomes

$$
\frac{\mathrm{d} W}{\mathrm{~d} x}=\alpha W^{-\sigma}(a W-b x), \quad \sigma=\frac{2-\alpha}{\alpha}>1,
$$

and then

$$
x \frac{\mathrm{d} y}{\mathrm{~d} x}=-(1+y)+b(a / b)^{\sigma+1} x^{1-\sigma}(1+y)^{-\sigma} y .
$$

Putting now $\eta=x^{\sigma-1}=x^{2(1-\alpha) / \alpha}$ we get

$$
(1-\sigma) \eta \frac{\mathrm{d} y}{\mathrm{~d} \eta}=-(1+y)+b(a / b)^{\sigma+1}(1+y)^{-\sigma} y / \eta
$$


An analysis of the linearized situation near $y=0, \eta=0$ allows to conclude that all orbits in our class satisfy the behaviour

$$
\lim _{\eta \rightarrow 0} \frac{y}{\eta}=k=\frac{b^{\sigma}}{a^{\sigma+1}}
$$

This means for the behaviour of $v$

$$
v^{\alpha}(x)=k_{0} x+k_{1} x^{\sigma}+\ldots
$$

with $k_{0}=b / a$. It can be checked on the expansions that the solutions are $C^{\infty}$ but not analytic. Integrating $\mathrm{d} x / v=-\mathrm{d} t$, we get

$$
x(t)=d_{1} t^{-\mu}+\ldots, \quad \mu=\frac{\alpha}{1-\alpha},
$$

and $d_{1}$ a function of $a, b$ and $\alpha$. Its precise value $d_{1}=(a / b)^{1 /(1-\alpha)} \mu^{\mu}$ can be easily computed from the first-order approximation

$$
a\left|x^{\prime}\right|^{\alpha-1} x^{\prime}+b x \sim 0
$$

which simplifies (1.3) and characterizes the behaviour of slow orbits near rest. Indeed, in slow orbits the inertial term represents a secondary effect.

\section{On the oscillating states}

As a conclusion of the foregoing analysis, we find that for $\alpha<1$ all orbits have a finite number of sign changes in their positive evolution. Moreover, solutions starting near the origin have one or no change sign, and these two situations can be precisely characterized by regions limited by the fast orbits $\Gamma_{*}$ and $\Gamma_{* *}$, which constitute the fast manifold $\Gamma$.

Thus, the region with no oscillations in the first and fourth quadrants is limited by $\Gamma_{*}$ on the right and the vertical axis on the left. A symmetric situation with $\Gamma_{* *}$ instead of $\Gamma_{*}$ happens in the second and third quadrants of the phase-plane $(x, v)$. This does not form a complete neighbourhood of the origin, some orbits near $(0,0)$ change sign.

The region of one sign-change is formed in Q-I, Q-IV by the region exterior to $\Gamma_{*}$ limited by the vertical axis and the continuation of $\Gamma_{* *}$ to those quadrants. A symmetric situation occurs in Q-II, Q-III.

The union of those regions completely surrounds the origin. Therefore, orbits near the origin change sign at most once.

The process of counting oscillations can be continued iteratively farther away from the origin.

\section{Singular DAmping, $\alpha \leq 0$}

\subsection{Coulomb friction}

We begin by reviewing the well-known case $\alpha=0$. The basic equation becomes

$$
v \frac{\mathrm{d} v}{\mathrm{~d} x}=a-b x, \quad v>0
$$

in the first and second quadrants, and

$$
v \frac{\mathrm{d} v}{\mathrm{~d} x}=-a-b x, \quad v<0,
$$


in the third and fourth. These equations can be integrated once to give

$$
\begin{cases}\frac{1}{2} v^{2}+\frac{b}{2}(x-L)^{2}=C, & v>0 \\ \frac{1}{2} v^{2}+\frac{b}{2}(x+L)^{2}=C, & v<0\end{cases}
$$

where $L=b / a$. The analysis of the fast orbit is still valid in the limit $\alpha \rightarrow 0$ putting in the calculations of previous section $\alpha=\beta=0, \gamma=1$, so that $w=v^{2}$ and $a_{1}=2 a$. We get the expressions for the fast orbit in the form $(2.12,2.13)$. Thus,

$$
v_{*}^{2}(t) \sim 2 a x+\cdots, \quad x_{*}(t) \sim(a / 2)\left(t_{0}-t\right)^{1 / 2}+\cdots
$$

There is a symmetric situation in the third quadrant, i.e., with $v$ and $x$ reversing signs.

However, the slow orbits are replaced in the limit by stabilization to a positive location $0<x_{\infty} \leq L$ in the first and fourth quadrants, and to the symmetric situation in the second and fourth, with exactly the same rate of approach (since they are the same orbits up to horizontal displacement in the $(x, v)$ plane).

It is easily observed that any orbit stops in a finite time at a point in the interval $-L \leq x \leq L$ after a finite number of oscillations.

\subsection{Case $\alpha<0$}

The basic equation becomes (with $\varepsilon=-\alpha$ ):

$$
v^{1+\varepsilon} \frac{\mathrm{d} v}{\mathrm{~d} x}=a-b x v^{\varepsilon}, \quad v>0
$$

and the symmetric situation for $v<0$ (just as before). The fast orbit is studied as before and has a similar expansion. The difference lies in its behaviour for large $x$ : it represents a particle that comes from $x=\infty$, takes on speed and then stops in finite time.

Instead of the slow orbits we obtain orbits that stop completely in finite time at any point $x_{\infty} \neq 0$. They have an analogous behaviour at $x=\infty$.

In all cases $\alpha \leq 0$ solutions may change sign only once, since the level $v=0$ is accepted only as a complete stop in finite time. On the other hand, when $\alpha>0$ complete stop in finite time happens only at $x=0$ and for the fast orbit.

\section{Study With a General Damping term}

We are now ready to attack the problem of existence of a fast orbit for the equation with a general damping term, equation (1.1). Here we do not assume that $f$ is a power, but only that $f$ is a real continuous function such that $f(s) s>0$ for $s \neq 0$ (so that $f(0)=0$ ). We also assume for convenience the smoothness $f \in C^{1}$ for $s \neq 0$. Later on, we will also need a small extra condition on the behaviour at $s=0$, for instance that $f$ is increasing in small neighbourhood. We will discuss the existence of a fast orbit in the first quadrant, i.e., for $x>0, v=-x^{\prime}>0$, and then only the values of $f$ for $s<0$ are involved. The discussion of the existence of a fast orbit for $x<0$ can be done under similar assumptions on the values of $f(s)$ for $s>0$ and need not be repeated.

\subsection{Formal analysis}

Equation (1.1) is written in the notation of Section 2 as

$$
v \frac{\mathrm{d} v}{\mathrm{~d} x}=-f(-v)-b x .
$$


Therefore, it is convenient to work with the function $\widehat{f}(s)=-f(-s)$. In order to study fast orbits we can make the same formal assumption as in Section 2 and solve

$$
x^{\prime \prime}(t)+f\left(x^{\prime}(t)\right) \sim 0, \quad v \frac{\mathrm{d} v}{\mathrm{~d} x} \sim \widehat{f}(v) .
$$

This gives the behaviour for orbits with $x>0, v=-x^{\prime}>0$ :

$$
t_{0}-t \sim \int_{0}^{v} \frac{\mathrm{d} v}{\widehat{f}(v)}, \quad x \sim \int_{0}^{v} \frac{v \mathrm{~d} v}{\widehat{f}(v)}
$$

Therefore, the conjecture is that the existence of a fast orbit in the first quadrant depends on the condition

$$
\int_{-v}^{0} \frac{\mathrm{d} s}{f(s)}=\int_{0}^{v} \frac{\mathrm{d} s}{\widehat{f}(s)}<\infty, \quad v>0
$$

(recall that $x^{\prime}=-v$ ). For power nonlinearities this conjecture coincides with the condition $\alpha<1$ that we have established above. But it also includes almost linear cases like

$$
f(s) \sim s(\log (1 /|s|))^{\beta} \quad \text { near } v=0,
$$

with $\beta>1$, or even $f(s) \sim s \log (1 /|s|)(\log \log (1 /|s|))^{\beta}$.

\subsection{Ruling out infinitely many oscillations}

We will first eliminate the possibility of stop in finite time for solutions which oscillate infinitely many times. This is a consequence of a time calculation which is best made in "radial coordinates", where we introduce the variables $r, \phi$ through

$$
b^{1 / 2} x=r \cos (\phi), \quad v=r \sin (\phi) .
$$

Then, the system $\mathrm{d} x / \mathrm{d} t=-v, \mathrm{~d} v / \mathrm{d} t=f(-v)+b x$ implies that

$$
\frac{\mathrm{d} \phi}{\mathrm{d} t}=b^{1 / 2}+\frac{1}{r} f(-r \sin \phi) \cos \phi,
$$

and the right-hand side is less than $b^{1 / 2}$ in the first and third quadrants. Therefore, completing a turn of $\pi / 2$ radians in those quadrants takes a time at least $\pi /(2 \sqrt{b})$, and the whole turn takes at least double of that time, i.e., we have

$$
T \geq \frac{\pi}{b^{1 / 2}}
$$

for each period. We conclude that finite-time stop orbits must necessarily be eventually monotone, and this can only happen in the first and third quadrants. Note that in the second quadrant $x<0$ and $\mathrm{d} x / \mathrm{d} t<0$, so that no orbit can approach the origin inside that quadrant. A symmetric situation occurs in the fourth quadrant.

\subsection{Topological considerations}

Before we address the question of the existence of a fast orbit we discuss the more general question of existence of a monotone orbit with $x>0$. Let us perform some preliminary analysis using only topological arguments in the phase-plane. By eventually monotone we mean that $x(t)$ and $v(t)=x^{\prime}(t)$ are positive for all $t \geq t_{0}$, i.e., it lives eventually in the first quadrant of phase plane. Immediate inspection says that the graphs of the orbits in 
the first quadrant are increasing in the region $R_{1}=\{\widehat{f}(v)>x\}$ and decreasing in $R_{2}=\{\widehat{f}(v)<x\}$. It is also clear that the only allowed transition as $x$ increases is from $R_{1}$ to $R_{2}$. Finally, any orbit that moves downwards towards zero in $x$ must eventually pass to region $R_{1}$ and then either it goes to $(0,0)$ or it goes over to the second quadrant.

We conclude from this analysis that once an eventually monotone orbit exists there exist infinitely many, i.e., all those lying below it in the first quadrant of the phase plane. In principle, for monotone $\widehat{f}$ there are three options: an eventually monotone orbit can go to zero in a fast or slow way, or it can stabilize to a point $x_{\infty}>0$. When $f(v) \rightarrow 0$ as $v \rightarrow 0$ the last situation cannot happen.

In order to check whether there exist eventually monotone orbits we use a shooting method: for every $a>0$ we solve the initial value problem

$$
v \frac{\mathrm{d} v}{\mathrm{~d} x}=\widehat{f}(v)-b x \quad v(0)=a
$$

to obtain a unique solution $v_{a}(x)$. This family is ordered, and moreover, each orbit is monotone in $K_{1}$, i.e., until it hits the line $\widehat{f}(v)=b x$. We may take the limit

$$
v_{\#}(x)=\lim _{a \rightarrow 0} v_{a}(x)
$$

Standard arguments show that only two options exist:

(i) the limit is defined in an interval $0 \leq x \leq x_{0}$ and then it is a positive and eventually monotone orbit. This is what happens for instance for supercritical linear damping and also in the power case with $\alpha<1$;

(ii) the family $\left\{v_{a}(x): a>0\right\}$ is defined on intervals that go to zero as $a \rightarrow 0$ so that no monotone solution exists in the limit. This is what happens for subcritical linear damping, as the reader will easily check.

In order to sort these cases we observe that once there exists a non-trivial limit in the above process for some nonlinearity $f_{1}$, there will be a nontrivial limit for all $f_{2}$ such that $\widehat{f}_{2}(v) \geq \widehat{f}_{1}(v)$ for $v$ near 0 . This follows from comparison of the direction fields in phase plane. Moreover, with obvious notations,

$$
v_{\#}\left(x ; f_{2}\right) \geq v_{\#}\left(x ; f_{1}\right) .
$$

We also have the following practical result to decide between options (i) and (ii), which follows from the linear friction case plus the previous topological analysis.

Proposition 4.1. There exists an eventually monotone orbit with $x, v>0$ going to $(0,0)$ if for instance

$$
\lim _{v \searrow 0} \frac{\widehat{f}(v)}{v} \geq c=\frac{a^{2}}{4 b} .
$$

The constant in the condition is sharp.

\subsection{Necessary condition}

We can now prove one part of our conjecture.

Theorem 4.2. Let $f$ be a real, continuous and negative function defined for $v \leq 0$, of class $C^{1}$ for $v<0$, and with $f(0)=0$. If there exists a fast positive orbit, then $f$ satisfies condition (4.4). Moreover, equation (4.3) holds in the form of inequalities:

$$
x \geq \int_{0}^{v} \frac{v \mathrm{~d} v}{\widehat{f}(v)}, \quad t_{0}-t \geq \int_{0}^{v} \frac{\mathrm{d} v}{\widehat{f}(v)}
$$


Proof. By hypothesis we have a fast orbit which means that the limit of the $v_{a}(x)$ is not trivial. Next, we compare the direction fields of our equation and

$$
v \frac{\mathrm{d} v}{\mathrm{~d} x}=\widehat{f}(v)
$$

Since for the latter the direction field is bigger we conclude that the corresponding orbits $v_{a}(x)$ are larger, hence the limit (let us call it $v_{1}(x)$ ) is larger, $v_{1}(x) \geq v(x)$, as long as both are defined and monotone. Hence, the orbit of (4.11) goes to zero with a higher velocity that $v$, and since this an orbit with fast rate of stabilization, so is $v_{1}(x)$. But $v_{1}(x)$ is given by exact integration of $v \mathrm{~d} v=\widehat{f}(v) \mathrm{d} x$, so that $x=F\left(v_{1}\right)$ with $F$ defined by

$$
F(v)=\int_{0}^{v} \frac{s \mathrm{~d} s}{\widehat{f}(s)} .
$$

We conclude that the integral must be finite. We also conclude easily that for the original orbit $v=v(x)$ we have $x \geq F(v)$.

Finally, using $t-t_{0}=\int_{0}^{x} \mathrm{~d} x / v$ and the previous estimate we get the second inequality:

$$
t_{0}-t=\int_{0}^{x} \frac{\mathrm{d} x}{v(x)} \geq \int_{0}^{x} \frac{\mathrm{d} x}{v_{1}(x)}=\int_{0}^{v_{1}} \frac{\mathrm{d} v}{\widehat{f}(v)} \geq \int_{0}^{v} \frac{\mathrm{d} v}{\widehat{f}(v)} .
$$

This completes the necessity part.

\subsection{The main result}

In order to obtain a necessary and sufficient condition we require $f$ to satisfy (4.4) plus a small condition on the behaviour at $v=0$. A condition of superlinear behaviour at $v=0-$, like $v / f(v) \leq C$ is sufficient. We can then prove our full conjecture.

Theorem 4.3. Assume that $f$ satisfies the above assumptions and is superlinear at $v=0-$. There exists a fast orbit if and only $f$ satisfies condition (4.4). Moreover, equation (4.3) holds:

$$
x=\int_{0}^{v} \frac{v \mathrm{~d} v}{\widehat{f}(v)}(1+o(v)), \quad t_{0}-t \geq \int_{0}^{v} \frac{\mathrm{d} v}{\widehat{f}(v)}(1+o(v)) .
$$

Proof. We only have to prove the sufficiency part. Under condition (4.4) we construct a fast monotone solution $v_{1}(x)$ of the equation

$$
v \frac{\mathrm{d} v}{\mathrm{~d} t}=(1-\varepsilon) \widehat{f}(v)
$$

We prove easily that for this orbit:

$$
\lim _{v \rightarrow 0} \frac{x}{\left.\widehat{f}\left(v_{1}\right)\right)}=\frac{1}{(1-\varepsilon)} \lim _{v_{1} \rightarrow 0} \frac{1}{\widehat{f}\left(v_{1}\right)} \int_{0}^{v_{1}} \frac{v \mathrm{~d} v}{\widehat{f}(v)}
$$

The last quantity goes to zero as $v_{1} \rightarrow 0$ since

$$
\frac{1}{\widehat{f}(v)} \int_{0}^{v} \frac{v \mathrm{~d} v}{\widehat{f}(v)} \leq \frac{v}{\widehat{f}(v)} \int_{0}^{v} \frac{\mathrm{d} v}{\widehat{f}(v)} \leq C \int_{0}^{v} \frac{\mathrm{d} v}{\widehat{f}(v)}
$$


and the last factor goes to zero as $v \rightarrow 0$ by the integrability assumption. This means that for small $x>0$ the function $v=v_{1}(x)$ is a subsolution to the original equation, that we write in the form

$$
\frac{v}{\widehat{f}(v)} \mathrm{d} v=\left(1-\frac{x}{\widehat{f}(v)}\right) \mathrm{d} x .
$$

Using the construction argument with $v_{a}$, we see that we are in option (i), so that an eventually monotone orbit $v_{\#}(x)$ exists for the original problem and $v_{\#}(x) \geq v_{1}(x)$. This also means that it is a fast orbit.

Remark 1. The extra condition is automatically satisfied if $f(s)$ is monotone nondecreasing for $s<0, s \sim 0$, and satisfies (4.4), since we have the following result:

Lemma 4.4. It $\int \mathrm{d} v / \widehat{f}(v)$ converges at $v=0$ and $\widehat{f}$ is monotone near $v=0$, then

$$
\lim _{v \rightarrow o} \frac{\widehat{f}(v)}{v}=\infty .
$$

The proof consists only of estimating in the range of monotonicity:

$$
\frac{c}{2 \widehat{f}(c)} \leq \int_{c / 2}^{c} \frac{\mathrm{d} v}{\widehat{f}(v)} \leq \int_{0}^{c} \frac{\mathrm{d} v}{\widehat{f}(v)},
$$

and the last quantity tends to zero as $c \rightarrow 0$ by the main integrability assumption. Next, we note that quasimonotonicity in the form $\widehat{f}^{\prime}(v) \geq-K \widehat{f}(v)$ is also sufficient to get the same conclusion. We have the following clearcut consequence:

Corollary 4.5. If $f$ is quasi-monotone near $v=0$, then there exists a fast orbit if and only $f$ satisfies condition (4.4).

Remark 2. A general additional condition that still guarantees the conclusion of the last theorem says that if we put $F(v)=\int_{0}^{v} \mathrm{~d} s / \widehat{f}(s)$, which is a continuous and increasing function, $C^{2}$ for $v>0$ under assumption (4.4), then we may ask that

$$
v F(v) F^{\prime}(v) \leq c<1
$$

for all $v$ small enough. Then the fast orbit exists.

Remark 3. Non-existence of fast orbits. As a consequence of the results, we have superlinear situations where fast orbits do not exist, for instance if

$$
\int_{0}^{v} \frac{\mathrm{d} v}{\widehat{f}(v)}=\infty, \quad \lim _{v \searrow 0} \frac{\widehat{f}(v)}{v}=\infty .
$$

Then all orbits can be classified as eventually oscillating or slow. This does not happen in the power case for $\alpha<1$. Actually, it extends the supercritical linear case.

Remark 4. The condition for the fast orbit in the third quadrant, i.e., with $x(t)<0$ and $x^{\prime}(t)=-v>0$, is similar:

$$
\int_{0}^{v} \frac{\mathrm{d} s}{f(s)}<\infty, \quad v>0 .
$$

We discuss now a serious question, what may happen when the assumptions are not fulfilled, in other words, we want to find the limits of the result. 
Remark 5. The sufficiency of condition (4.4) is false if no extra behaviour at zero (like monotonicity or superlinearity) is imposed. Here is a counterexample. It is easy to construct an $\widehat{f}$ satisfying (4.4) that has infinitely many local minima $v_{n}$ accumulating at $v=0$ with $\widehat{f}\left(v_{n}\right) \sim 0$, such that for every $n$ there is an orbit crossing the line $x=\widehat{f}(v)$ at $v \sim v_{n}$ that passes to the second quadrant (it oscillates). Then monotone orbits cannot exist. Hint: start with an example where $\widehat{f}\left(v_{n}\right)=0$. Then make a small perturbation iteratively for $n=1,2, \ldots$

Remark 6. The same happens if $f(s) s$ takes on negative values in any lateral neighbourhood of $s=0$ (depending on whether we look for positive or negative fast orbits). Hence, the sign condition for $f$ is essential at least near the origin. Otherwise, no monotone solutions can exist.

The case when $f$ is bounded away from zero. We have assumed that $f$ is continuous at $v=0$ with $f(0)=0$. In order to generalize the Coulomb case and the power cases with $\alpha<0$ we may assume that $\widehat{f}(v)$ is bounded from below away from zero $(\widehat{f}(v) \geq c>0)$ as $v \rightarrow 0, v>0$. Then there is necessarily one fast orbit, the rest are orbits that stop at other points. The proof is an easy adaptation of Section 3 plus the topological arguments to this situation. We refrain from giving more details on this issue.

\section{Fast orbits in Other CONTEXts}

\subsection{Fast orbits in nonlinear diffusion}

We have obtained a criterion that solves the question of existence of fast orbits, i.e., those that correspond to a nonlinearly damped oscillator that stops in finite time. It is remarkable that the same mathematical condition on the nonlinear function determines the validity of the property of finite speed of propagation in filtration equations of the type

$$
u_{t}=(\phi(u))_{x x} \quad \text { or } f(v)_{t}=v_{x x}
$$

where $\phi$ is monotone increasing function, $f=\phi^{-1}$ is its inverse and we put $v=\phi(u), u=f(v)$. The condition, exactly (4.16), appears in the work of Oleinik et al. [20] in 1958 and the related questions have been extensively studied since then, see $[2,9]$.

It is worth commenting the idea on which the similarity of results relies in such different problems. Indeed, the characterization of the property of finite propagation for equation (5.1) is performed by reducing it to the existence of a certain type of travelling waves, namely those that reach the level $u=v=0$ at a finite distance. A travelling wave is a solution of the form

$$
u(x, t)=U(s), \quad s=x-c t
$$

with $c \neq 0$, say $c>0$. The equations for $U$ and for $V=\phi(U)$ are:

$$
\phi(U)^{\prime \prime}+c U^{\prime}=0, \quad V^{\prime \prime}+c f(V)^{\prime}=0
$$

where the prime denotes derivative with respect to $s$. We want to obtain solutions $U(s)$ which are positive in an interval, say $0 \leq s<s_{0}$ and vanish at $s_{0}$ with slope $\phi(U)^{\prime}=V^{\prime}=0$ so that they are continued as $U=0$ for $s>s_{0}$ (at least as weak solutions). In other words, we look for a fast orbit for $V=\phi(U)$. The equation for $V$ resembles the two first terms of equation (1.1) but not exactly, because of the different form of the last term. Indeed, integrating once we get

$$
V^{\prime}=c f(V)
$$

from which the standard condition of fast orbit follows as the condition for a correct travelling wave. If we want to see the similarity of equations we have to introduce the variable $W$ such that $W^{\prime}=V$ and then the last 
equation is written as

$$
W^{\prime \prime}-c f\left(W^{\prime}\right)=0
$$

Up to trivial changes this equation is the same as the first terms of (1.1), which are the ones involved in determining the fast orbit. By the way, $W$ can be directly obtained by looking for travelling waves of the so-called gradient-dependent nonlinear diffusion equation

$$
u_{t}=\left(\phi\left(u_{x}\right)\right)_{x}
$$

An even more direct similarity happens for the so-called dual porous medium equation,

$$
u_{t}=\phi\left(u_{x x}\right)
$$

Of course, these equations are closely related. In all cases the condition of finite propagation, which is often called preservation of compact support, is given by

$$
\int_{0} \frac{\mathrm{d} v}{f(v)}<\infty, \quad \int_{0} \frac{\phi^{\prime}(u)}{u} \mathrm{~d} u<\infty .
$$

\subsection{Comments on the dynamical classification}

The idea of the 3-fold alternative (slow - fast - oscillatory) is also well known in nonlinear diffusion, particularly in the study of the class of special solutions called self-similar solutions. We refer to $[6,18]$ among many other papers. It is a beautiful mathematical result that deserves to be better known.

It is important to note that the above 3-way alternative is not the only that appearing in this type of framework. Thus, a different 3-way alternative appears in the study of travelling waves for the equation

$$
u_{t}=\left(u^{m}\right)_{x x}-u^{\alpha}
$$

with $\alpha<1$. There, the profiles reach zero in 3 ways that represent advancing and receding waves, separated by one stationary wave, $c f$. [12-14]. If we perform the TW analysis like in the previous section, we will see that the equations obtained are 3-term variations of (1.1), but in this case they cannot be reduced to it.

In another example, self-similar profiles of $u_{t}=\left(u^{m-1} u_{x}\right)_{x}$ can be classified in the same way (slow, fast or oscillatory orbits) if $m>0$, but the classification becomes slow - fast - superfast if $m \geq 0$, so-called very fast diffusion, cf. [11].

The use of asymptotic methods has a long story in dynamics, both for ODEs and also for PDEs. For instance, the pioneering work of Prandtl in boundary layer theory in hydrodynamics at the beginning of the $20^{\text {th }}$ century combines both subjects. The early work was formal mathematics and a large part of work on complex engineering problems still is, but rigorous development is now on sure foot in many basic examples. We have applied here some of the basic ideas to solve the problem of stabilization of a nonlinearly damped oscillator. We refer to classical texts like [7,15,21] for more material on ODEs, normal forms, stable, unstable and center manifolds, etc.

As for blow-up techniques, a very beautiful analysis of blow-up of a singularity happens in the study of focusing solutions of the porous medium equation. This has been studied by Aronson and collaborators [1,4,5], and extended to other models in $[3,8]$.

Work partially supported by the European IHP network "Hyperbolic and Kinetic Equations: Asymptotics, Numerics, Applications", RNT2 2001349. 


\section{REFERENCES}

[1] S. Angenent and D.G. Aronson, The focusing problem for the radially symmetric porous medium equation. Comm. Partial Differential Equations 20 (1995) 1217-1240.

[2] D.G. Aronson, The Porous Medium Equation. Springer-Verlag, Berlin/New York, Lecture Notes in Math. 1224 (1985).

[3] D.G. Aronson, O. Gil and J.L. Vázquez, Limit behaviour of focusing solutions to nonlinear diffusions. Comm. Partial Differential Equations 23 (1998) 307-332.

[4] D.G. Aronson and J. Graveleau, A selfsimilar solution to the focusing problem for the porous medium equation. Euro. J. Appl. Math. 4 (1992) 65-81.

[5] D.G. Aronson and J.L. Vázquez, The porous medium equation as a finite-speed approximation to a Hamilton-Jacobi equation. Ann. Inst. H. Poincaré Anal. Non Linéaire 4 (1987) 203-330.

[6] H. Brezis, L.A. Peletier and D. Terman, A very singular solution of the heat equation with absorption. Arch. Rational Mech. Anal. 95 (1986) 185-209.

[7] J. Carr, Applications of centre manifold theory. Springer-Verlag, New York-Berlin, Appl. Math. Sci. 35 (1981) vi+142 pp.

[8] M. Chaves and V. Galaktionov, On the focusing problem for the PME with absorption. A geometrical approach (in preparation).

[9] J.I. Díaz, Nonlinear partial differential equations and free boundaries. Vol. I. Elliptic equations. Pitman (Advanced Publishing Program), Boston, MA, Res. Notes in Math. 106 (1985).

[10] J.I. Díaz and A. Liñán, On the asymptotic behaviour for a damped oscillator under a sublinear friction. Rev. Acad. Cien. Ser. A Mat. 95 (2001) 155-160.

[11] R. Ferreira and J.L. Vázquez, Self-similar solutions to a very fast diffusion equation. Adv. Differential Equations (to appear).

[12] V.A. Galaktionov, S.I. Shmarev and J.L. Vázquez, Second-order interface equations for nonlinear diffusion with very strong absorption. Commun. Contemp. Math. 1 (1999) 51-64.

[13] V.A. Galaktionov, S.I. Shmarev and J.L. Vázquez, Behaviour of interfaces in a diffusion-absorption equation with critical exponents. Interfaces Free Bound. 2 (2000) 425-448.

[14] V.A. Galaktionov, S.I. Shmarev and J.L. Vázquez, Regularity of interfaces in diffusion processes under the influence of strong absorption. Arch. Ration. Mech. Anal. 149 (1999) 183-212.

[15] J. Guckenheimer and Ph. Holmes, Nonlinear oscillations, dynamical systems, and bifurcations of vector fields. Revised and corrected reprint of the 1983 original. Springer-Verlag, New York, Appl. Math. Sci. 42 (1990).

[16] A. Haraux, Comportement à l'infini pour certains systèmes non linéaires. Proc. Roy. Soc. Edinburgh Ser. A 84 (1979) $213-234$.

[17] M.W. Hirsch and S. Smale, Differential equations, dynamical systems, and linear algebra. Academic Press, New York-London, Pure Appl. Math. 60 (1974).

[18] S. Kamin, L.A. Peletier and J.L. Vázquez, A nonlinear diffusion-absorption equation with unbounded initial data, in Nonlinear diffusion equations and their equilibrium states, Vol. 3. Gregynog (1989) 243-263. Birkhäuser Boston, Boston, MA, Progr. Nonlinear Differential Equations Appl. 7 (1992).

[19] E.B. Lee and L. Markus, Foundations of Optimal Control Theory. J. Wiley and Sons, New York, SIAM Ser. Appl. Math. (1967).

[20] O.A. Oleinik, A.S. Kalashnikov and Y.-I. Chzou, The Cauchy problem and boundary problems for equations of the type of unsteady filtration. Izv. Akad. Nauk SSR Ser. Mat. 22 (1958) 667-704.

[21] L. Perko, Differential equations and dynamical systems, Third edition. Springer-Verlag, New York, Texts in Appl. Math. 7 (2001).

[22] J.L. Vázquez, An Introduction to the Mathematical Theory of the Porous Medium Equation, in Shape Optimization and Free Boundaries, edited by M.C. Delfour. Kluwer Ac. Publ., Dordrecht, Boston and Leiden, Math. Phys. Sci. Ser. C 380 (1992) 347-389. 